\title{
The Negative Non-Finite Form of Albanian and the Way it is Expressed in Latin
}

\author{
Dr. Leonard Xhamani \\ Docente di Latino, Università di Tirana \\ E-mail: leonardxhamani@gmail.com
}

\section{Doi:10.5901/mjss.2014.v5n8p616}

\section{Abstract}

During its historical development Albanian language has created not only finite forms but also non-finite forms, one of them being the negative non-finite form. The verb in this non-finite form indicates that an action - mainly in the past or the future - is not performed. Scholars of Albanian have given various opinions in terms of how this structure is constructed. However, the authoritative source, the book published by the Academy of Sciences of Albania (Gramatikë e gjuhës shqipe), gives the following definition: The negative non-finite form is structured by putting the negative particle "without" in front of the participle of the verb: e.g. pa pasur (without having), pa menduar (without thinking), pa vënë (without putting), etc. Even though it has five non-finite forms, Latin does not have such a structure as without + participle, as in Albanian. Latin, on the other hand, has a corresponding structure: sine + the ablative of the noun that corresponds to the verb, for example: pa u druajtur (without hesitating) = sine timore, pa bërtitur (without shouting) = sine clamore; pa qarë (without crying) = sine lacrimis, etc. However, the Latin structure that comes closer to the Albanian negative non-finite form is the participle preceded by the negative in the form of an apposition or the absolute ablative, for instance: pa qenë $i$ kërkuar (without being searched) = non rogatus; pa qenë $i$ thirrur (without being called) $=$ non vocatus, etc.

Keywords: Albanian language, Latin language, negative non-finite form.

Il verbo in questa forma implicita esprime il mancato compimento dell'azione specialmente nel passato oppure nel futuro. Gli studiosi della lingua albanese hanno dato pareri differenti concernenti la modalità di formazione di questa forma. Sheperi (2001: 88) afferma "il participio diventa negativo con la preposizione pa". Domi (1952: 215) lo nomina "participio accompagnato dalla particella pa", mentre nella pagina 230 I chiama "participio accompagnato dalla preposizione pa". Myderrizi (1944: 128) esprime l'idea che il participio diventa negativo essendo preceduto dall'avverbio pa. L'ultima parola spetta comunque al libro dell'Accademia dove si può leggere: "La forma implicita negativa si costruisce premettendo al participio del verbo la particella pa: pa pasur, pa qenë, pa menduar, pa vënë, pa ngrënë ecc." (Akademia e Shkencave, Instituti i Gjuhësisë dhe i Letërsisë: 301). Una tale forma implicita non è conosciuta nella lingua latina, ma, come vedremo di seguito, a questa forma corrispondono altre costruzioni. Entrambi gli elementi di questa costruzione analitica sono unificati, sia dal punto di vista semantico, sia grammaticale (morfologico e sintattico), eppure la particella pa conserva tuttavia il significato della negazione, che nasce dalla sua provenienza come preposizione che esprime carenza e più generalmente negazione. Così nella frase: U zgjua pa zbardhur, questa forma vuole dire "kur ende nuk kishte zbardhur".

Qualche volta nell'uso si scontra anche una forma del tipo pa pasë larë, pa pasë shkuar, la quale viene costruita premettendo al participio del verbo la forma implicita negativa dell'ausiliare kam (pa pasë). L'esistenza di queste forme indica che la costruzione del tipo pa + pjesore të paparanyjëzuar è una forma verbale implicita di un tipo particolare, poiché il cosiddetto participio passato pasë shkuar, non esiste. Questa forma sovracomposta verrà chiamata il passato della forma implicita negativa. In contrapposizione a questa, la forma del tipo pa larë si può chiamare convenzionalmente il presente. Tenendo conto che il participio come parte della frase si usa più raramente, perché è stato sostituito dall'aggettivo verbale (Agalliu, 1972: 55) e serve per di più come parte integrante di alcune forme verbali analitiche e sintetiche, cioè come elemento costruttivo, dimostra che la forma implicita pa + pjesore të paparanyjëzuar differisce dal participio. Questa forma è di un tipo particolare e non ha corrispondenti in altre lingue.

In questa forma implicita sono intrecciate le caratteristiche del verbo e dell'avverbio (Çeliku, 1986: 93). Essa ha un significato semantico e alcune particolarità grammaticali che lo distinguono dal participio. I verbi attivi transitivi quando si usano in questa forma implicita, hanno regolarmente significato attivo, mentre nel participio hanno significato passivo. Come conseguenza i verbi transitivi usati in questa forma implicita hanno la possibilità di reggere anche complementi diretti o indiretti, espressi non soltanto con sostantivi e pronomi differenti, ma anche con particelle dei pronomi personali. Anche i verbi intransitivi in questa forma implicita possono reggere complementi indiretti, espressi anche con le particelle dei pronomi personali. Per esempio: 
$\Leftrightarrow$ Nuk do të iki pa ju treguar për miqtë e mi. Nuk lashë vend pa të kërkuar.

$\Leftrightarrow$ Nëna nuk linte rast pa ua demonstruar me mburrje vizitorëve, ... (Kongoli, Jetë në një kuti shkrepësesh, f. 33).

$\leftrightarrow$ Kishin ardhur rresht, për të vajtuar një femër të plagosur, pa e ditur se ishin rrëzuar vetë ndërkaq. U tërhoq për t’u lënë vendin të tjerëve dhe, pa i shkuar mendja se i kishte duart me dhe, fshiu mollëzat. (Kadare, Spiritus, f. $139,148)$.

Mentre il participio non ha nessuna categoria grammaticale, la forma implicita pa larë ha la categoria della diatesi. I verbi transitivi in questa forma implicita si usano non solo nella diatesi attiva, ma anche in altre diatesi, le quali si costruiscono introducendo la particella $u$ tra la particella pa ed il participio.

$\Leftrightarrow$ Ajo rrinte shtrirë pa u shqetësuar nga zhurmat.

$\Leftrightarrow$ Megjithëse bënte çmos t'i kryente veprimet mëngjesore pa u ndier, mua më dilte gjumi. E mbante në dhomën e tij deri në mëngjes, pa u kujdesur së paku të mos e shihnin fëmijët. (Kongoli, Jetë në një kuti shkrepësesh, f. $57,113)$.

Inoltre essa ha un significato negativo condizionato dal significato temporale del verbo reggente ed esprime il mancato compimento dell'azione che doveva essere verificato nel passato oppure che si aspetta verificarsi nel futuro, di solito prima dell'azione nominata dal verbo reggente. Indica, cioè anteriorità in relazione al verbo reggente.

$\Leftrightarrow$ Pa luajtur këmbët, nuk luajnë dhëmbët.

Questa forma implicita, a differenza del participio, può avere anche un soggetto differente da quello del verbo reggente.

$\Leftrightarrow$ Ai u ngrit herët, pa zbardhur dita.

$\Leftrightarrow$ Pa mbaruar java, u bë një mbledhje e ngutshme në Komitetin e Partisë. (Kadare, Darka e gabuar, f. 108).

Per quanto riguarda la costruzione del tipo verbo ausialiare kam oppure jam + forma implicita negativa non si può parlare di grammaticalizzazione. Le costruzioni con il presente e l'imperfetto di questi verbi sono sinonimiche con il passato, rispettivamente il trapassato prossimo dell'indicativo nella forma negativa (Agalliu, 1983: 116). Cosi:

$\leftrightarrow$ kishin dy muaj pa u parë = s'ishin parë prej dy muajsh

$\Leftrightarrow$ ishte pa u përgatitur = nuk ishte përgatitur

Queste costruzioni hanno il significato della forma attiva, quando derivano da verbi intransitivi, e quello della diatesi passiva, quando derivano da verbi transitivi.

In queste costruzioni il verbo jam ha la funzione della copula, conserva, cioè, il suo significato dell'essere e non è trasformato e nemmeno ha intrapreso la via del passaggio in verbo ausiliare. Cioè, dal punto di vista sintattico questa costruzione verrà trattata come predicato nominale e non verbale. Con i verbi del tipo mbetem, la forma implicita negativa risulta più evidente nel ruolo del predicato, come membro della frase, il cui significato si collega nello stesso tempo al soggetto 0 al complemento e al verbo: qëndruam pa folur, e la pa prekur. Per esempio:

$\leftrightarrow$ jë copë herë të gjatë qëndruan pa folur, secili në tryezën e vet. (Kadare, Spiritus, f. 143).

\section{Le funzioni sintattiche della forma implicita negativa}

1. Nella funzione del determinante predicativo dopo i verbi: jam, kam, mbetem, rri, lë, ecc.

$\Leftrightarrow$ Ai iu afrua miqve që ishin akoma pa u ulur në vendet e tyre.

$\Leftrightarrow$ Për momentin mbeti si dru pa lëvizur.

$\stackrel{\longmapsto}{\longrightarrow}$ Një copë herë të gjatë qëndruan pa folur, secili në tryezën e vet. (Kadare, Spiritus, f. 143).

2. Nella funzione del predicato accanto ad un soggetto espresso con sostantivo o con pronome personale. La possibilità del funzionamento delle forme implicite in qualità di predicato è diversa. Questo si collega specialmente con il significato che esprime la costruzione con forma implicita (Instituti i Gjuhësisë dhe i Letërsisë, 1997: 179). Tra le forme implicite nella funzione del predicato di più si scontra quella negativa.

$\leftrightarrow$ Pa mbaruar fjalën unë, në dhomë hyri babai.

$\stackrel{\leftrightarrow}{*}$ Pa hyrë pranvera mirë, pemët ishin mbushur me lule.

$\Leftrightarrow$ Pa mbaruar java, u bë një mbledhje e ngutshme në Komitet të Partisë. (Kadare, Darka e gabuar, f. 108).

Nel ruolo di un predicato, essa può avere un soggetto tutto suo, anzi si può allargare anche con un complemento, complemento di luogo oppure proposizione subordinata complementare.

Pa hyrë në oborr dëgjoi të lehurat e qenit.

$\stackrel{\leftrightarrow}{\hookrightarrow}$ Pa mbërritur mirë në zyrë, bashkë me kafenë e mëngjesit i sillnin aparatin. (Kadare, Spiritus, f. 154).

3. Nella funzione del complemento di tempo. In questa funzione, la forma implicita negativa ha il significato dell'anteriorità. L'azione del verbo principale si compie prima dell'azione espressa da questa forma implicita. Per 
determinare se si tratta di anteriorità oppure no, prenderemo in considerazione l'azione del verbo reggente, poiché è proprio quest'azione che viene determinata dalla forma implicita negativa (Çeliku, 1986: 95).

${ }_{\rightarrow}$ Pa dalë ende nga porta kryesore, ku Remzi Kadareja bëhej gati t'u thoshte diçka, përfundimi i parë i hetimit ishte marrë vesh. (Kadare, Darka e gabuar, f. 126).

$\stackrel{\leftrightarrow}{\longrightarrow}$ Në mëngjes u ngritën që pa dalë drita.

La forma implicita negativa si può accompagnare anche con un avverbio.

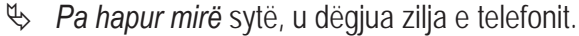

$\Leftrightarrow$ Pa thënë asgjë, ajo e mbylli derën. (Kadare, Spiritus, f. 202).

Nella funzione del complemento di tempo, questa forma implicita può anche apparire separata.

$\stackrel{\leftrightarrow}{\rightarrow}$ Sot, që pa gdhirë, u grabit një bankë.

4. Nella funzione del complemento di modo. In questa funzione, la forma implicita negativa caratterizza l'azione del verbo reggente, indicando la modalità di compimento.

$\Leftrightarrow$ Atë betejë e fitoi pothuajse pa derdhur gjak.

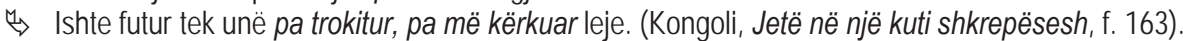

$\leftrightarrow$ Telefoni kishte një copë herë që po binte, por ai e ngriti dhe e mbylli dorezën pa folur. Pa i hapur sytë, Arian Vogli dëgjonte zërin e gruas përmes frymëmarrjes që vinte e shpeshtohej. (Kadare, Spiritus, f. 144, 148).

Quando questa forma implicita si usa nella funzione del complemento di modo si nota un'avverbializzazione, cioè il passaggio ad un avverbio (Hysa, 1970: 49 \& Demiraj, 1970: 130) quali: papritur, padashur, papandehur, papushuar, pakuptuar, ecc. II passaggio di queste forme in avverbi è avvenuto come risultato del loro uso frequente accanto ad un altro verbo, nella funzione simile a quella dell'avverbio.

$\Leftrightarrow$ Pasdite u sëmur papritur.

$\Leftrightarrow$ Nga pozicioni ku ishte, qëllonte parreshtur.

$\Leftrightarrow$ Përfytyroni një prokuror të shtetit që papritur pajiset me parashtesën ish. (Kongoli, Jetë në një kuti shkrepësesh, f. 179).

$\Leftrightarrow$ Shpend Guraziu preku padashur çelësat. (Kadare, Spiritus, f. 129).

$\leftrightarrow$ Në vend që ta dëgjonte tjetrin, fare padashur përfytyronte monologun e Kaligulës me kalin e vet konsull. (Kadare, E penguara, f. 48).

Si può notare che, quando si trasformano in avverbi, queste forme subiscono modifiche anche nella scrittura, cioè, si scrivono insieme. Per qualche avverbio oppure per alcuni sintagmi in via di avverbializzazione completa, si notano alcune forme che indicano che essi non sono completamente cristallizzati (Çeliku, 2006: 182). Così pakuptuar si usa con il significato degli avverbi vetiu, padashur e, solitamente, si scrive come un insieme:

$\stackrel{\leftrightarrow}{\hookrightarrow}$...krejt si pakuptuar kishin ikur ndërkaq tre vjet nga vdekja e tij. (Kadare, Spiritus, f. 164).

5. Nella funzione del complemento condizionale. Quando la forma implicita negativa si usa in questa funzione, il verbo reggente è negativo. La sua negazione, dunque, si esprime tramite una particella negativa (mos, s', nuk).

$\leftrightarrow$ Pa luajtur këmbët, nuk luajnë dhëmbët.

$\leftrightarrow$ Pa ngrënë hudhra, s'të vjen erë goja.

II verbo reggente può essere affermativo solo nelle frasi interrogative con significato negativo.

$\Leftrightarrow$ Si do të ikje pa u takuar me mua?

Le relazioni di condizione in casi simili sono nate avendo come base le relazioni temporali della posteriotià. Prima si compie l'azione della forma implicita negativa, poi l'azione principale (Çeliku, 1986: 100).

$\Leftrightarrow$ Pa u marrë vesh me miqtë e tu, nuk do të ikësh.

\section{"Pa" seguito da una parola in lingua latina}

La forma implicita dell'albanese, del tipo pa + participio, nella lingua latina non esiste, ma si può dare con queste forme (Tantucci, 1992: 370; Ghiselli \& Concialini, 2006: 180)

a) Con sine e l'ablativo del sostantivo che corrisponde al verbo: pa u druajtur = sine timore; pa bërtitur = sine clamore; pa qarë = sine lacrimis ecc.

$>$ Sed nullus ordo, nullum imperium certum, cum suo quisque consilio uteretur atque omnes sine timore iter facerent...

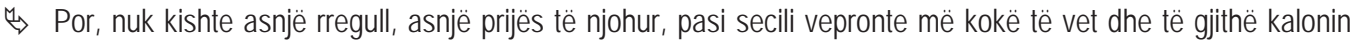
nga një rrugë në tjetrën, pa pasur frikë... (Caes. Liber I, kap. 51). 
$>$ Qui inopinantes pabulatores et sine ullo dissipatos timore aggressi magnum numerum iumentorum...

$\leftrightarrow$ Këta sulmojnë në befasi ushtarët e foragjereve të Afranit, që ishin shpërndarë sa andej-këtej, pa pasur asnjë frikë dhe u rrëmbejnë një numër të madh kafshësh barre... (Caes. Liber I, kap. 55).

$>$ Caesar in eam spem venerat, se sine pugna et sine vulnere suorum rem conficere posse, quod re frumentaria adversarios interclusisset.

$\Leftrightarrow$ Çezari shpresonte ta përfundonte sipërmarrjen pa luftë dhe pa plagosur asnjë prej ushtarëve të vet, sepse mendonte se ua kishte ndërprerë furnizimin kundërshtarëve. (Caes. Liber I, kap. 72).

$>$... nova exoptant, odio suarum rerum mutari omnia student, turba atque seditionibus sine cura aluntur, quoniam egestas facile habetur sine damno.

$\leftrightarrow$... lakmojnë të renë, përpiqen të ndërrojnë gjithçka, duke mos duruar gjendjen e vet, mëkohen me turbullirat dhe kryengritjet, pa u kujdesur për asgjë, sepse kush është i varfër, s'ka ç'të humbasë.

$>$ Dicam sine cunctatione quod sentio (Cic.)

$\Leftrightarrow$ Do të them pa ngurruar atë që mendoj.

b) Con un aggettivo dal significato negativo: pa dashur = invitus; pa e ditur = ignarus, inscius; pa e pritur = imprūdens; pa u njohur = incognittus; pa folur = tacĭtus ecc. In alcuni casi questi aggettivi si traducono in albanese con sostantivi oppure con aggettivi. Per esempio:

> Cuius cognito consilio Caesar frustra diebus aliquot consumptis, ne reliquum tempus amittat, infectis eis, quae agere destinaverat, ab urbe proficiscitur atque in ulteriorem Galliam pervenit.

$\leftrightarrow$ Si kuptoi qëllimin e tij, pasi kishin kaluar kot disa ditë, por për të mos humbur më kohë tjetër, Çezari del nga Roma, pa kryer punët që kishte vendosur të bënte dhe drejtohet në Galinë Ulteriore. (Caes. Liber I, kap. 33).

$>$ Eis autem invitis, a quibus Pharus tenetur, non potest esse propter angustias navibus introitus in portum.

$\Leftrightarrow$ Pa pëlqimin e tyre, që mbanin Farin, anijet nuk mund të hynin në port, falë ngushtësisë së hyrjes së tij. (Caes. Liber III, kap. 112).

$>$ Ne quid eis noceatur, neu quis invitus sacramentum dicere cogatur, a Caesare cavetur.

$\leftrightarrow$ Çezari u jep fjalën se askush prej tyre nuk do të prekej dhe se askush nuk do të bënte betimin ushtarak, pa e dashur. (Caes. Liber I, kap. 86).

> Interim L Nasidius, a Cn. Pompeio cum classe navium XVI, in quibus paucae erant aeratae, L. Domitio Massiliensibusque subsidio missus, freto Siciliae imprudente atque inopinante Curione pervehitur...

$\leftrightarrow$ Ndërkaq, Luc Nasidi, i dërguar nga Pompeu në mbështetje të Luc Domicit dhe të marsejezëve me një flotë prej 16 anijesh, disa prej të cilave të veshura me pllaka bronzi, kapërcen ngushticën e Siçilisë, duke e zënë Kurionin në befasi dhe pa e pritur... (Caes. Liber II, kap. 3).

> Latum ab $\mathrm{X}$ tribunis plebis contradicentibus inimicis, Catone vero acerrime repugnante et pristina consuetudine dicendi mora dies extrahente, ut sui ratio absentis haberetur...

$\leftrightarrow$ Dhjetë tribunët e popullit propozuan, megjithëse armiqtë kundërshtuan, megjithëse Katoni kundërshtoi ashpër dhe pavarësisht zakonit të tij të vjetër për ta shtyrë ditën me diskutime pa fund, që kandidatura e tij të merrej parasysh pa qenë i pranishëm... (Caes. Liber I, kap. 32).

$>$ Agesilāus satrăpas regios imparatos imprudentesque offendit.

$\Leftrightarrow$ Agelisao i befasoi satrapët mbretërorë pa qenë të përgatitur dhe pa e pritur.

c) Con un participio preceduto dalla negazione nella funzione di un'apposizione oppure in ablativo assoluto : pa qenë i kërkuar = non rogatus; pa qenë i provokuar = non lacessitus; pa qenë i thirrur = non vocatus; pa dyshuar $=$ non dubitans; pa pas përmbyllur asgjë = rebus infectis; pa shkelur rregullat = salvis legibus; pa ngurruar, pa u vonuar = nullā interpositā morā. Soltanto questa forma può corrispondere alla forma implicita del tipo pa + participio dell'albanese. Per esempio:

$>$ Haec cum in Achaia atque apud Dyrrachium gererentur, Scipionemque in Macedoniam venisse constaret, non oblitus pristini instituti Caesar mittit ad eum A. Clodium...

$\leftrightarrow$ Ndërsa në Akaia dhe pranë Dyrrahut zhvilloheshin këto ngjarje dhe, nga burime të sigurta, ishte mësuar se Skipioni kishte mbërritur në Maqedoni, pa harruar propozimin e vet të vjetër, Çezari nisi tek ai Klodin... (Caes. Liber III, kap. 57).

> Neque ibi constitit, sed eadem celeritate, paucos suos ex fuga nactus, nocturno itinere non intermisso, comitatu equitum XXX ad mare pervenit...

$\leftrightarrow$ Edhe atje nuk qëndroi, por vazhdoi me të njëjtën shpejtësi dhe, duke hasur gjatë ikjes në ca njerëz të vet, pa e ndalur udhëtimin as natën, arriti buzë detit, i shoqëruar nga 30 kalorës... (Caes. Liber III, kap. 96).

$>$ Illi non diu commorati nec longius ab infimo colle progressi copias in castra reducunt. 
$\leftrightarrow$ Ata, pa u vonuar shumë dhe pa përparuar më tepër se deri te rrëza e kodrës, i kthyen trupat në shatorre. (Caes. Liber I, kap. 42).

$>$ Recepto Caesar Orico nulla interposita mora Apolloniam proficiscitur.

$\Leftrightarrow$ Si shtiu në dorë Orikumin, pa u vonuar, Çezari niset për në Apoloni. (Caes. Liber III, kap. 12).

$>$ Itaque nulla interposita mora sauciorum modo et aegrorum habita ratione impedimenta omnia silentio prima nocte ex castris Apolloniam praemisit.

$\leftrightarrow$ Kështu, pa u vonuar, si siguroi të sëmurët dhe të plagosurit, sapo filloi të ngrysej, në heshtje të plotë... (Caes. Liber III, kap. 75).

$>$ Non haesitans respondēbo (Cic.).

$\Leftrightarrow$ Do të përgjigjem pa ngurruar.

$>$ Fabius, nec die nec nocte intermisso itiněre, ad exercitum rediit (Liv.)

$\Leftrightarrow$ Fabio, pa ndërprerë marshimin as ditë as natë, u kthye tek ushtria.

d) Con una proposizione coordinativa con quella che precede con nec, neque. In casi simili il verbo collegato con questa congiunzione, si può esprimere anche con una forma implicita. Per esempio:

$>$ Illi non diu commorati nec longius ab infimo colle progressi copias in castra reducunt.

$\leftrightarrow$ Ata, pa u vonuar shumë dhe pa përparuar më tepër se deri te rrëza e kodrës, i kthyen trupat në shatorre. (Caes. Liber III, kap. 42).

$>$... nec minimam partem temporis equitum vim cetrati sustinere potuerunt omnesque ab eis circumventi in conspectu utriusque exercitus interficiuntur.

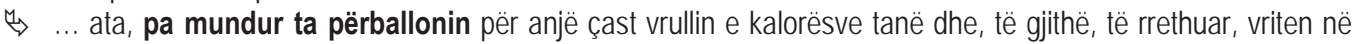
prani të të dyja ushtrive.

$>$ Postremo omnes aut de honoribus suis aut de praemiis pecuniae aut de persequendis inimicitiis agebant nec, quibus rationibus superare possent, sed, quemadmodum uti victoria deberent, cogitabant.

$\leftrightarrow$ Në fund të fundit, të gjithë përpiqeshin ose për të siguruar detyrat publike që ëndërronin, ose për shpërblime në të holla, ose për t'u hakmarrë ndaj armiqve personalë, pa menduar si të arrinin fitoren, por si të nxirrnin përfitime nga fitorja. (Caes. Liber III, kap. 83).

$>$ Rapimur in errorem nec vera cernimus (Cic.).

$\Leftrightarrow$ E lëmë veten të biem në gabim pa dalluar të vërtetën.

e) con una proposizione dipendente preceduta da cum non, ut non, oppure, soltanto dopo le proposizioni negative, da quin (o qui non) ed il congiuntivo.

> Parte circiter tertia exercitus eo biduo dimissa duas legiones suas antecedere, reliquas subsequi iussit, ut non longo inter se spatio castra facerent.

$\leftrightarrow$ Pasi liroi një të tretën e ushtrisë Brenda dy ditëve të para, urdhëroi dy nga legjionet e veta që të ecnin në krye, të tjerëve u dha urdhër t'i ndiqnin pas, pa lënë shumë hapësirë... (Caes. Liber I, kap. 87).

$>$ His rebus tantum fiduciae ac spiritus Pompeianis accessit, ut non de ratione belli cogitarent, sed vicisse iam viderentur.

$\stackrel{4}{\rightarrow}$ Pas këtyre ngjarjeve, popmejanët u mbushën me besim e krenari, pa u shqetësuar për vazhdimin e luftës, por e shihnin veten fitimtarë. (Caes. Liber III, kap. 72).

$>$ Quod se facturos minabantur, aegreque tunc sunt retenti, quin oppidum irrumperent, graviterque cam rem tulerunt...

$\leftrightarrow$ Këtë kërcënonin se do të bënin, prandaj mezi janë përmbajtur pa u sulur në qytet dhe me shpirt të thyer e duruan këtë gjë... (Caes. Liber III, kap. 13).

$>$ Treviri nullum tempus intemisērunt quin legatos mittěrent (Ces.)

$\Leftrightarrow$ Trevirët nuk lanë të kalonte kohë pa ftuar ambasadorët.

$>$ Possum pedes movēre ut non curam (Sen.)

$\Leftrightarrow$ Mund të lëviz këmbët pa vrapuar.

Come conclusione possiamo dire che il verbo in questa forma implicita esprime il mancato compimento di un'azione specialmente nel passato oppure nel futuro. La forma implicita negativa si costruisce premettendo al participio la particella pa: Entrambi gli elementi di questa costruzione analitica sono unificati dal punto di vista semantico, nonché grammaticale (morfologico e sintattico). In questa forma implicita sono intrecciate le caratteristiche del verbo e dell'avverbio.

I verbi transitivi usati in questa forma implicita hanno la possibilità di reggere anche complementi diretti o indiretti. In questa forma implicita anche i verbi intransitivi possono reggere complementi indiretti espressi con le particelle dei pronomi personali. 
I verbi transitivi in questa forma implicita si usano nella diatesi attiva e passiva (pa bërë - pa u bërë). In alcuni casi in questa forma si nota una certa contraddizione di carattere temporale del tipo pa pasë bërë, la quale si contrappone alla forma semplice del tipo pa bërë che, senza dubbio è anche la più usata. Questa forma sovracomposta verrà chiamato passato della forma implicita negativa, mentre la forma del tipo pa bërë si può chiamare convenzionalmente tempo presente.

La forma implicita negativa si usa nella funzione del complemento predicativo dopo i verbi: jam, kam, mbetem, rri, lë, ecc; nella funzione di un predicato accanto ad un soggetto espresso con un sostantivo oppure con un pronome personale; nella funzione del complemento di tempo; nella funzione del complemento di modo; nella funzione del complemento condizionale; serve per esprimere la mancanza di un'azione secondaria, che dal punto di vista del significato si presenta coordinato con quella del verbo reggente.

La forma implicita dell'albanese, del tipo pa + participio, nella lingua latina non esiste, ma si può dare con queste forme: con sine e l'ablativo del sostantivo che corrisponde al verbo: pa u druajtur = sine timore; pa bërtitur = sine clamore; pa qarë = sine lacrimis ecc; con un aggettivo dal significato negativo: pa dashur = invitus; pa e ditur = ignarus, insčus; pa e pritur = imprūdens; pa u njohur = incognitus; pa folur = tacitus ecc; con un participio preceduto dalla negazione oppure in ablativo assoluto: pa qenë i kërkuar = non rogatus; pa qenë i thirrur = non vocatus; pa dyshuar = non dubitans; pa pas përmbyllur asgjë = rebus infectis; pa shkelur rregullat = salvis legibus; pa u vonuar = nullā interpositā morā; con una proposizione coordinata a quella che precede con nec, neque; con una proposizione dipendente preceduta da cum non, ut non, oppure, soltanto dopo le proposizioni negative da quin (oppure qui non) ed il congiuntivo.

\section{References}

Agalliu Fatmir (1983), Vëzhgime mbi formën gramatikore pa + pjesore, SF, 3, Tiranë.

Akademia e Shkencave, Instituti i Gjuhësisë dhe i Letërsisë (1997), Gramatika e gjuhës shqipe, II, Tiranë.

Caesar Caius Julius (2008), De bello civili, Torino: UTET.

Çeliku Mehmet (1986), Kuptimet dhe përdorimet sintaksore të trajtës së tipit pa + punuar në gjuhën e sotme letrare shqipe, SF, 3, Tiranë.

Çeliku Mehmet (2006), Format e pashtjelluara të foljes në gjuhën e sotme shqipe, Tiranë: Shblu.

Demiraj Shaban (1962), Morfologjia e gjuhës së sotme shqipe, pjesa II, Tiranë: Mihal Duri,

Demiraj Shaban (1970), Rreth ndajfoljëzimit të disa formave gramatikore dhe të disa togjeve të tipit parafjalë + emër, SF, 3, Tiranë.

Diotti Angelo (2000), Lexis 2, Roma: Bruno Mondadori.

Domi Mahir (1952), Gramatika e gjuhës shqipe, pjesa e dytë, sintaksa, Tiranë.

Ghiselli Alfredo, Concialini Gabriella (2006), II nuovo libro di latino, Bari: Laterza.

Hysa Enver (1970), Ndajfoljet në gjuhën e sotme shqipe, Studime Filologjike, 3, Tiranë.

Kadare Ismail (1996), Spiritus, Tiranë: Onufri.

Kadare Ismail (2008), Darka e gabuar, Tiranë: Onufri.

Kadare Ismail (2009), E penguara, Tiranë: Onufri.

Kongoli Fatos (2009), Jetë në një kuti shkrepësesh, Tiranë: Toena.

Myderrizi Osman (1944), Gramatika e re e shqipes, Tiranë.

Piazzino C., Fragonara A. (1985), Humanitatis magistra, Torino: Paravia.

Sallustius Caius Crispus, (2008), Catilinae coniuratio, Milano: Oscarmondadori.

Sheperi llia Dilo (1927), Gramatika dhe sintaksa e gjuhës shqipe, Vlorë: Shtypshkronja Vlora.

Tantucci Vittorio (1992), Urbis et orbis lingua, Bologna: Poseidonia. 